\title{
Article
}

\section{Laccase Redox Potentials: pH Dependence and Mutants - a QM/MM Study}

\author{
Jan Philipp Götze, and Michael Buehl
}

J. Phys. Chem. B, Just Accepted Manuscript • DOI: 10.1021/acs.jpcb.6b04978 • Publication Date (Web): 17 Aug 2016

Downloaded from http://pubs.acs.org on August 19, 2016

\section{Just Accepted}

"Just Accepted" manuscripts have been peer-reviewed and accepted for publication. They are posted online prior to technical editing, formatting for publication and author proofing. The American Chemical Society provides "Just Accepted" as a free service to the research community to expedite the dissemination of scientific material as soon as possible after acceptance. "Just Accepted" manuscripts appear in full in PDF format accompanied by an HTML abstract. "Just Accepted" manuscripts have been fully peer reviewed, but should not be considered the official version of record. They are accessible to all readers and citable by the Digital Object Identifier (DOI@). "Just Accepted" is an optional service offered to authors. Therefore, the "Just Accepted" Web site may not include all articles that will be published in the journal. After a manuscript is technically edited and formatted, it will be removed from the "Just Accepted" Web site and published as an ASAP article. Note that technical editing may introduce minor changes to the manuscript text and/or graphics which could affect content, and all legal disclaimers and ethical guidelines that apply to the journal pertain. ACS cannot be held responsible for errors or consequences arising from the use of information contained in these "Just Accepted" manuscripts. 


\title{
Laccase Redox Potentials: pH Dependence and Mutants - A QM/MM Study
}

Jan P. Götze and Michael Bühl*

EastChem School of Chemistry, University of St Andrews, North Haugh, St Andrews, Fife, KY16 9ST, UK Fax: 01334 463800; Tel: 01334 467235;

E-mail:mb105@st-andrews.ac.uk

\begin{abstract}
We have studied the $T$. versicolor laccase T1 site redox potential (RP) at the M06/6$311++G^{* *} / \mathrm{SDD}(\mathrm{Cu})$ level of theory, employing QM/MM optimised geometries (RI-BP86/def2SVP/def2-TZVP(Cu):CHARMM) of the whole protein system with electronic embedding. The oxidation state of the trinuclear cluster was found to affect the T1 site RP by about 0.2-0.3 V, depending on the protein protonation state. The computed laccase RP can be drastically lowered upon introduction of a protonation state corresponding to a neutral environment, by up to $-1.37 \mathrm{~V}$, which is likely an overestimation of the effect in vivo. The gradual change of the protonation state by single points without optimisation or equilibration results in a change that is even larger, namely up to about $-2.6 \mathrm{~V}$. Thus, the preferred protein conformation supports a high redox potential, compensating for the RP-lowering effect of surface charges. The predicted change in RP on going to the F463M mutant, ca. $-0.1 \mathrm{~V}$, is consistent with observations for a related laccase. Based on our results, we also propose and test a D206N mutant, but find it to be locked in a conformation with slightly lower RP.
\end{abstract}

\section{Introduction}

Lignin is a global source of biomass, attracting increasing attention as sustainable chemical feedstock. Extremely resistant toward degradation, its valorisation is proving highly challenging. In nature, lignin is attacked and degraded by fungi and other organisms to access the valuable cellulose and hemicellulose compounds. Lignin is a protective heterogenous polymer lacking distinct motifs that can be exploited for easy disassembly. It is believed that its degradation is triggered by redox reactions mediated by enzymes that have evolved for this purpose. These enzymes have been shown, among others, to catalyse the reaction of lignin model compounds to form oxidized radicals. ${ }^{1,2}$ As lignin-degrading activity has been related to the oxidative power of these enzymes, their redox potentials are of great fundamental and potentially practical interest. ${ }^{3}$

Laccase is a multicopper oxidase (MCO), and represents a special case in the group of the lignindegrading enzymes. ${ }^{2}$ Many ligninolytic enzymes employ heme as their active group and belong to the family of peroxidases. ${ }^{1,4}$ Laccase uses $\mathrm{O}_{2}$ (instead of $\mathrm{H}_{2} \mathrm{O}_{2}$ for peroxidases) as sink for the electrons obtained from the oxidation of the target redox mediator. ${ }^{5}$ As molecular oxygen is a very cheap resource, laccase is very attractive for technical applications. ${ }^{6}$

MCOs in general have found a lot of attention as targets for computational modelling over the recent years. The stepwise changes in redox potential during $\mathrm{O}_{2}$ activation and effects of groups close to the redox centres have been studied on a quantum chemical level. ${ }^{4,7,8} \mathrm{~A}$ recent article even lists the individual steps and relative redox potentials for wild type CueO, ${ }^{9}$ a well-studied MCO. ${ }^{8,10}$ The MCO reaction is split between two spatially separated $\mathrm{Cu}$ centres; the mono-Cu T1 centre being the one responsible for oxidation of the target compounds, and the trinuclear Cu centre (TNC) being 
the one reducing $\mathrm{O}_{2}$ (Figure 1). ${ }^{11} \mathrm{~T} 1$ centres are found in several (non-MCO) enzymes not featuring a $\mathrm{TNC}^{12}$ for example in plastocyanin. ${ }^{13}$ Until today, most MCO-related computational articles have been studying either the T1 centres or the TNC only, ${ }^{14,15}$ apart from a few exceptions. ${ }^{9}$

In the cases where both sites were present, the articles focused on the coupling between the two. ${ }^{9,16}$ Electron transfer between the sites has been associated to large unoccupied orbitals spanning the gap between them; and they have been found to affect each other's redox potential depending on the redox state present. The electronic coupling has been related to the hydrogen bond network, as it may serve as a direct connection between the two sites. ${ }^{5}$

While computational approaches have helped to elucidate the reaction mechanism and the internal relative redox potentials of the wild type, there have been no studies so far actually mimicking experimental measurements by providing a standard method that works well for both the laccase active sites as well as for a common redox standard such as the ferrocene/ferrocenium ( $\left.\mathrm{Fc} / \mathrm{Fc}^{+}\right)$ couple. As we have computed such relative redox potentials (RRPs) previously for other heme-based systems, ${ }^{4,7}$ we now extend these studies to laccase. In this context, we were interested in the effect

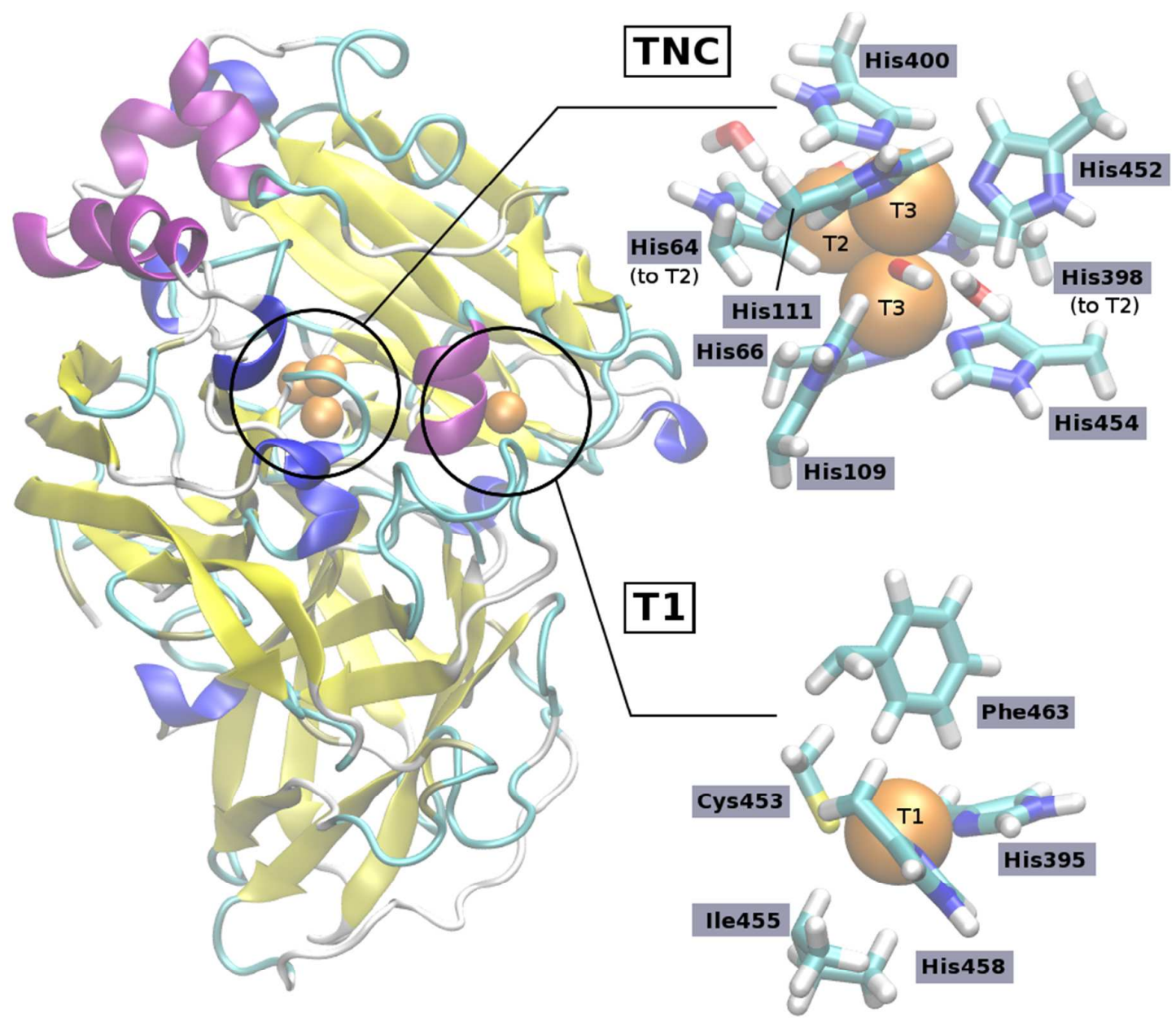

Figure 1 Trametes versicolor laccase from PDB entry $1 \mathrm{GYC}^{11}$ with trinuclear cluster (TNC) and copper sites (T1, T2 and T3) highlighted. The T1 site shown corresponds to the QM region employed in the QM/MM calculations for the wild type protein. Image created using VMD. ${ }^{17}$ 
of long-range electrostatic effects, focusing on the $\mathrm{T} 1$ site, arising from the protein protonation state as well as the charge state of the TNC. Note that we diverge from other approaches for computing the redox potentials proposed elsewhere, ${ }^{9,18}$ as we have acquired the RRPs of another lignilolytic enzyme before with an analogous protocol within the desired level of accuracy. ${ }^{4}$ For quantitative precision, one would require to perform, for example, extended free-enery perturbation simulations. For our qualitative approach here, however, we use fewer snapshots, but allow for some flexibility of the active centres.

We thus seek to answer the following questions: (i) Is the redox potential of the T1 site dependent on explicit coupling to the TNC? It has been shown recently that the TNC can affect the T1 centre redox potential, ${ }^{9}$ but it is unclear to what extent this effect can be reproduced in a model explicitly containing only the $\mathrm{T} 1$ site. (il) How sensitive is laccase towards the environmental pH? As this can be systematically studied in silico, we adapted the protein protonation states to a neutral and a low$\mathrm{pH}$ environment. (iii) What effect does a F463M mutant have on the T1 site in laccase? The F463M mutant protein of Trametes versicolor laccase is resulting in a structure analogous to plant laccases or the laccase CotA from Bacillus subtilis. ${ }^{2}$ The "inverse" CotA M463F mutant shows a RP that is $0.097 \mathrm{~V}$ higher than the wild type RP, and we seek to reproduce this result for a similar change in laccase. ${ }^{19}$

\section{Methods}

\section{Ferrocene standard calibration}

The density functional employed for the remainder of this study, $\mathrm{M} 06,{ }^{20}$ was determined via a series of test calculations employing a Born-Haber $\mathrm{cycle}^{7,21} \mathrm{for} \mathrm{Fc} / \mathrm{Fc}^{+}$and a complex mimicking the laccase T1 active site (from the Cambridge Crystal Structure Database entry DEDXER; ${ }^{22}$ redox couple<smiles></smiles>

Chart 1 Structure of the employed reference redox complex $\left[\mathrm{Me}_{2} \mathrm{NN}\right] \mathrm{CuSCPh}\left(\mathrm{CSD}\right.$ entry DEDXER, “D”). ${ }^{20}$

abbreviated as " $\mathrm{D} / \mathrm{D}^{\prime}$ ", Chart 1$)$. The detailed results of the method calibrations can be found in the Supplementary Information (SI); a full description of the calibration procedure can be found elsewhere. ${ }^{7}$ The calculations were performed using the Gaussian09 software. ${ }^{23}$

The procedure involves the optimisation and subsequent thermochemistry corrections with the $B P 86^{24,25}$ functional, which we have validated previously for geometries of transition metal 
complexes, ${ }^{26}$ and which has performed well in our earlier studies of RRPs. ${ }^{4,7}$ Previous articles on $\mathrm{MCOs}^{8,9}$ have reported $\mathrm{PBE}^{27,28}$ to be the method of choice, others ${ }^{29,30}$ promoted $\mathrm{PBEO}^{31}$ when reproducing the redox potential of model complexes. To allow for direct comparison between different systems, we decided to stay with our established procedures of using BP86 for optimisation and finding a functional suitable for the evaluation of the redox potential via single points. For the optimisation and normal mode analyses, we employed RI-BP86 with the basis set to be used for the $\mathrm{QM} / \mathrm{MM}$ optimisation (def2-SVP, with def2-TZVP for Fe and $\mathrm{Cu}$, " $\mathrm{E}_{\text {small }}$ ). ${ }^{32,33}$ The final electronic energy is obtained from a single point employing a variety of functionals involving a larger augmented Pople basis $\left(6-311++G^{* *} \text {, " } \mathrm{E}_{\text {large }}\right)^{34}$ plus the Stuttgart/Dresden effective core potential (SDD) for the transition metal ( $\mathrm{Cu}$ in case of $\mathrm{D}^{-} / \mathrm{D}$ or $\mathrm{Fe}$ in case of $\left.\mathrm{Fc} / \mathrm{Fc}^{+}\right)^{35}$ in a $\mathrm{CPCM}$ model mimicking tetrahydrofuran (THF; $\varepsilon=7.4257$ ). ${ }^{36,37}$ This procedure is performed for both the reduced and oxidised states of the redox couple in question $\left(\mathrm{Fc} / \mathrm{Fc}^{+}\right.$or $\left.\mathrm{D}^{-} / \mathrm{D}\right)$.

The absolute redox potential (ARP) for the couple then is computed via

$E_{A R P}=E_{\text {large, } C P C M}$ (Oxidised $)-E_{\text {large, CPCM }}$ (Reduced)

For small model systems, free energy corrections can be introduced from the harmonic vibrational frequencies computed in the gas phase

$\Delta \mathrm{G}=\mathrm{G}_{\text {small }}-\mathrm{E}_{\text {small }}$

Here, $G_{\text {small }}$ is free energy of the system obtained from a normal mode analysis (with thermal corrections at $298.15 \mathrm{~K}$ and $1 \mathrm{bar}$ ) and $\mathrm{E}_{\text {small }}$ is the optimised potential energy. For BP86, the contribution is $+0.15 \vee$ to the redox potential $\left(+0.10 \vee\right.$ for $\mathrm{D}^{-} / \mathrm{D},-0.05 \mathrm{~V}$ for $\mathrm{Fc} / \mathrm{Fc}^{+}$, see $\left.\mathrm{SI}\right)$. For comparison, we additionally built a model system that mimics the laccase $T 1$ site better than $D / D$, namely $\mathrm{Cu}(\mathrm{Im})_{2}(\mathrm{SMe})(\mathrm{Im}=$ imidazole, $\mathrm{Me}=$ methyl) with $\mathrm{Cu}(\mathrm{I}) / \mathrm{Cu}(\mathrm{II})$ centres. At the BP86/def2SVP/def2-TZVP(Cu) level, the contribution to the relative redox potential from these thermodynamic corrections is $0.11 \mathrm{~V}(+0.06 \mathrm{~V}$ contribution from the ARP of the small model, see $\mathrm{SI})$. As such we expect our calculations for the enzyme system to slightly overestimate the actual RRP, as we calculate them from the differences in the electronic energies only

$E_{R R P}=E_{A R P}\left(D^{-} / D\right)-E_{A R P}\left(F c / F c^{+}\right)$

Unfortunately, there are no experiments available that measure directly against the (IUPAC) standard of $\mathrm{Fc} / \mathrm{Fc}^{+}$in the case of $\mathrm{D}^{-} / \mathrm{D}$. However, the RRP to $\mathrm{Fc} / \mathrm{Fc}^{+}$can be obtained by a series of shifts (for example, from standard calomel electrode to $\mathrm{Fc} / \mathrm{Fc}^{+}$in THF). ${ }^{38}$ Hence, the experimental reference value for the RRP of $\mathrm{D}^{-} / \mathrm{D}$ vs. $\mathrm{Fc} / \mathrm{Fc}^{+}$in THF is located at $-0.98 \mathrm{~V}$ (shifted from $-0.18 \mathrm{~V} \mathrm{vs}$. $\mathrm{SHE}){ }^{22}$ As already noted above, $\mathrm{MO6}^{20}$ was found to be most accurate out of all tested methods, with BP86 following second; both functionals were thus chosen for the study of the enzyme. To allow for the direct comparison to other studies, we also provide a dataset employing the PBE functional in the SI.

As we also report the absolute redox potentials, one might be interested in comparing to an absolute reference value of laccase. Taking $4.28 \mathrm{~V}$ as a standard for the $\mathrm{SHE}^{39}, 40$ and $0.78 \mathrm{~V}$ for the enzyme ${ }^{41}$ the ARP of the investigated system should be at about $5.06 \mathrm{~V}$. As we have employed RRPs in our earlier studies, we will stick to the discussion of RRPs where appropriate. 


\section{Laccase structure and models}

Laccase from Trametes versicolor (Protein data bank entry $1 \mathrm{GYC}{ }^{11}$ was used as the model system for our study and sampled via Charmm27 molecular dynamics (MD) simulations. ${ }^{42}$ Missing hydrogen atoms were added beforehand using the Charmm hbuild function, except for $\mathrm{Cys} 460\left(\mathrm{H}_{\psi}\right)$, which was omitted to allow a thiolate coordination to the $\mathrm{T} 1 \mathrm{Cu}$ centre. All Lys and Arg residues were always considered to be protonated, as well as the usually deprotonated Asp77 close to the Laccase TNC cluster to prevent a strong interaction with a TNC hydroxyl group (data not shown). ${ }^{2}$ Histidine residues were either positively charged (\#55, 91, 153, 192, 306, 377 and 402) or protonated in either $\delta$ - (\# 64, 109, 111, 216, 398, 400, 452 and 454) or ع-positions (\# 66, 133, 395 and 458), depending on their hydrogen bonding environment or involvement in Cu-coordination. His98 is located on the surface and was thus either doubly or $\delta$-protonated, analogous to the surfaceaccessible acidic residues in the model (see below). Also, the crystal water oxygen bound to the $\mathrm{Cu}$ ion coordinated by His64 and His398 is in all cases represented as a hydroxide ion. This is in agreement with TNC redox states that were previously studied elsewhere ${ }^{9}$ (see below for details). We used the remaining titratable residues to generate two protein protonation states: Several Glu and Asp residues were adapted to a neutral or low-pH environment, if they were not crucial for neutralising a nearby charge via a salt bridge. In all cases, Asp77 remained protonated as indicated above, and residues 18, 96, 118, 131, 138, 140, 150, 214, 224, 288, 381, 419, 424, 469, 473 and 486 were kept deprotonated, i.e. negatively charged. The remaining $20 \mathrm{Glu}$ and Asp residues (see the gradual deprotonation section below for a full list) were varied to obtain the two models, either being negatively charged, resulting in the "neutral" environment model, or protonated, resulting in the "low-pH" environment model. As these 20 residues were almost exclusively located on the surface of the protein, we will refer to them in further discussions as "surface charges" (see SI, "Surface charge location").

For each of the two protonation states, we further created two variants, each representing a different TNC redox state. One model aims to recreate the native intermediate ("NI") of laccase, corresponding to the fully oxidised state of the TNC. Note that this requires the presence of an oxide ion $\left(\mathrm{O}^{2-}\right)$ located in the centre of the TNC. Furthermore, a crystal oxygen bound between the T3 copper ions is represented as a hydroxide ion for the NI MD simulations. The other MD simulation aimed at the state that contains a fully reduced TNC ("Red"). The Red state contains no oxide ion, only one TNC hydroxyl ion, and all TNC $\mathrm{Cu}$ ions are in the reduced $\mathrm{Cu}(\mathrm{I})$ state. During all MD simulations, the $\mathrm{T} 1 \mathrm{Cu}$ was in the oxidised $\mathrm{Cu}(\mathrm{II})$ state. From the $\mathrm{NI}_{\mathrm{low}-\mathrm{pH}}$ state starting geometry, we also created an F463M mutant protein, which was subject to the same treatment as the other variants. This variation was introduced due to the knowledge from analogous $B$. subtilis CotA mutants that it should shift the redox potential by about $0.1 \mathrm{~V} .{ }^{19}$ With this mutant protein, we obtain a set of five models $\left(\mathrm{NI}_{\text {neutral, }} \mathrm{NI}_{\text {low-pH }}, \operatorname{Red}_{\text {neutral, }}, \operatorname{Red}_{\text {low- } \mathrm{pH}}, \mathrm{NI}_{\text {low-pH/F463M }}\right)$. Note that we assigned the "NI" and "Red" labels in analogy to work presented by Li and coworkers. ${ }^{9}$ From the optimised structure of

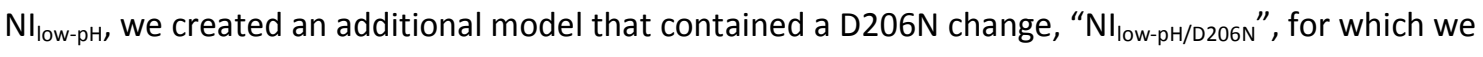
explored the effect of a asparagine residue instead of a protonated aspartate close to the T1 copper centre.

Molecular dynamics 


\section{TNC $\quad \mathrm{H} 2 \mathrm{O}$}
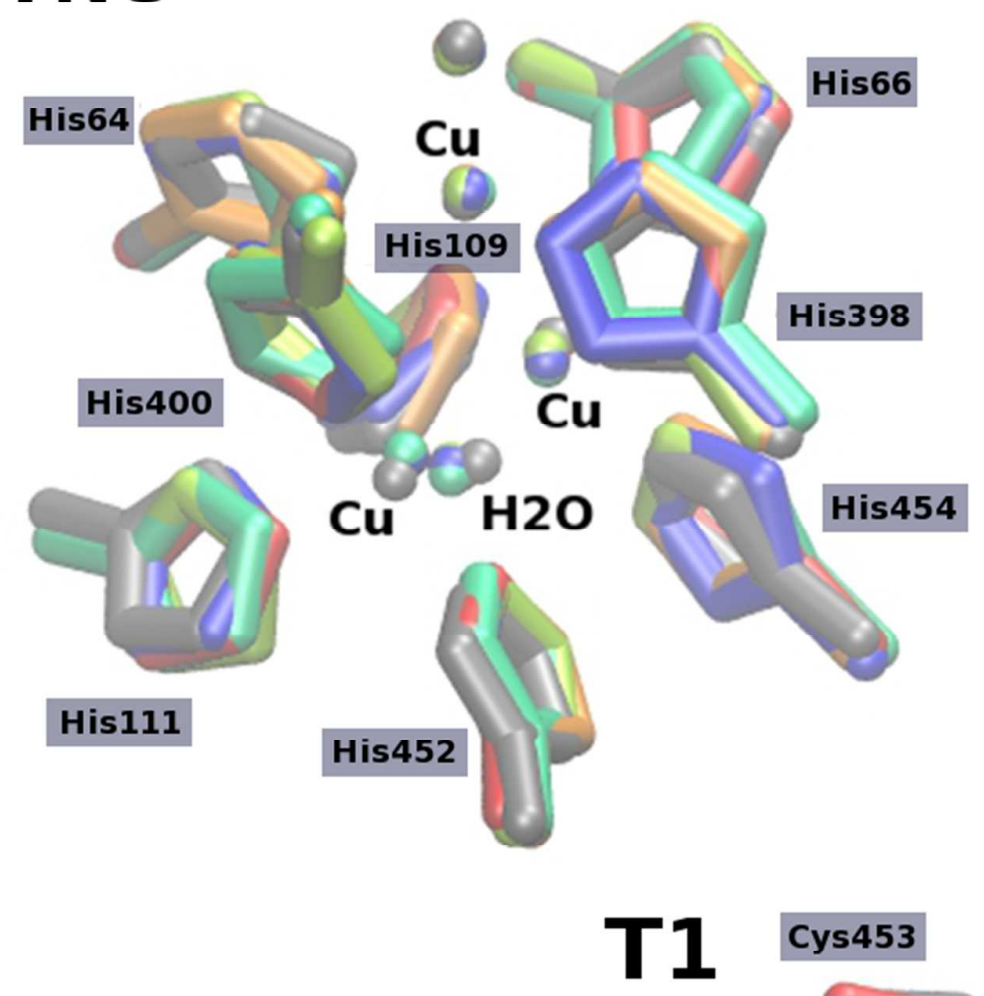

Ile455

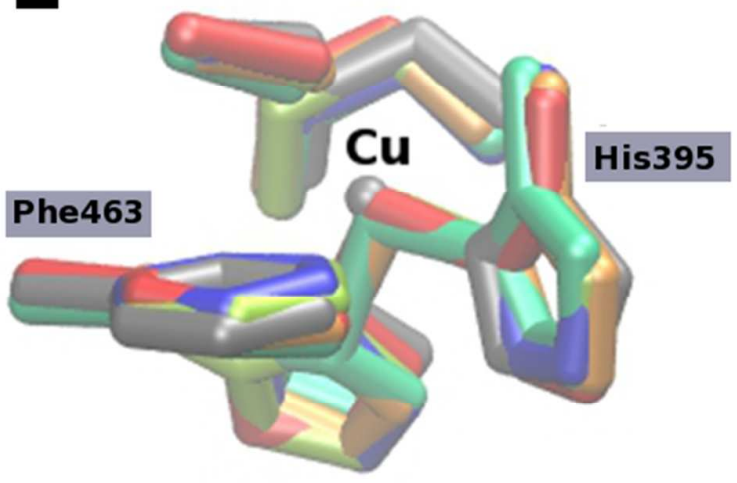

His458

Figure 2 Overlay of five MD snapshots, taken every 100 ps from the $\operatorname{Red}_{\mathrm{low}-\mathrm{pH}} \mathrm{MD}$ trajectory, only showing the nonhydrogen atoms of the $\mathrm{Cu}$ coordination spheres in T1 and TNC. Crystal structure shown as grey, snapshots as colours (red, orange, yellow, green, blue), after RMSD alignment. Note that the QM region (T1 site) also exhibits only small conformational changes.

After steepest descent optimisation of the hydrogen atoms, the system was solvated in a water sphere of $35 \AA$ radius, centred on the T1 Cu site. The system was then charge neutralised by sodium or chloride ions, with the exact amount depending on the reduction and protonation state, explicitly $1 \mathrm{Na}^{+}$for the "neutral" models and $20 \mathrm{Cl}^{-}$for all others. The difference of 21 charges arises from the 20 reprotonated acidic residues plus the change in the protonation state of His98. Ions were generated by randomly replacing water molecules from a preliminary protonation shell using $\mathrm{VMD},{ }^{17}$ 
with a minimum distance of $4 \AA$ to the protein. Following a 100 ps NVT dynamics at $300 \mathrm{~K}$ with $1 \mathrm{fs}$ time step, the solvation/equilibration procedure was repeated four additional times. Throughout all simulations containing the water sphere, a harmonic boundary potential was imposed to keep the sphere intact. The protein is slightly larger than the employed sphere; any atoms outside of the sphere boundaries were kept frozen at all times after hydrogen optimisation.

Before releasing the protein inside the water sphere to generate the MD production trajectories, harmonic restraints along selected internal degrees of freedom (bonds and bond angles) had to be constructed to keep the $\mathrm{Cu}$ complexes intact. Especially for the TNC, the $\mathrm{Cu}(\mathrm{II})-\mathrm{Cu}(\mathrm{II})$ Coulomb interactions will otherwise break the system. Simple Cartesian freezing of atoms would have been not sufficient, as the two clusters (T1 and TNC) need to be able to change their relative orientation. For all employed force field restraints, the harmonic constants were set to $500 \mathrm{kcal} \mathrm{mol}^{-1} \AA^{-2}$. The full list of employed bonds and angles can be found in the provided file archive and in the SI; a pictorial representation for the $\mathrm{T} 1$ site of the wild type can be found in the $\mathrm{SI}$ as well. The effect of the restraints on the MD simulation is shown in Figure 2; it can be seen that the system is able to move but keeps its overall structure intact and close to the crystal structure. To obtain the force field parameters, we performed three pairs of hybrid quantum mechanics/molecular mechanics (QM/MM) optimisations, alternating between the T1 or TNC Cu cluster on the BP86/def2-SVP/def2$\operatorname{TZVP}(\mathrm{Cu})$ level of theory while keeping the non-QM cluster frozen (using the solvated $\mathrm{NI}_{\text {neutral }}$ model as basis). Starting geometry for each subsequent optimisation was the final point of the previous run treating the other cluster in the QM region. The QM regions of these calculations included the $\mathrm{Cu}$ ions of the target cluster, all coordinating residues and (in the case of TNC) the oxide/hydroxyl ions (non-solvated protein, starting from the crystal structure geometry) to obtain reference values. The actual calculation parameters were identical to the production $\mathrm{QM} / \mathrm{MM}$ models (see below), only the QM region switched back and forth between TNC and T1. The resulting geometries did not change anymore after the second iteration and were used to set up the force field restraints of all models. Note that we used some NI model parameters for the Red model (see SI) and Figure 2 shows that the parameters are suited for the Red models as well; however, since the electronic properties of the TNC are never explicitly taken into account, we consider this approach to be valid. To check if the TNC properties have a strong influence on our T1 Cu RP, we have computed the RP on the basis of a TNC using integer charges or charges derived from natural orbitals in a TNC QM/MM test calculation, and we found the results to differ by $<0.1 \mathrm{~V}$ (data not shown).

The actual production trajectories were started following a full optimisation of the whole system (under the spherical boundary conditions and $\mathrm{Cu}$ cluster restraints indicated above). They were performed in a NVT setup with a $1 \mathrm{fs}$ time step, heating the system stepwise to $300 \mathrm{~K}$ by $1 \mathrm{~K}$ per ps, then equilibrating for $200 \mathrm{ps}$ and then running the simulation for an additional $1000 \mathrm{ps}$. Those last 1000 ps were taken as the basis for our QM/MM analysis, with one snapshot every 100 ps starting at the 100 ps mark. In addition, 10 ns trajectories were produced for the WT and the D206N mutant (using the same MD set-up and starting from the pre-equilibrated structure at $0 \mathrm{ps}$ with a different random seed).

\section{QM/MM optimisations}

The ten snapshots of each MD simulation were used as initial points for $\mathrm{QM} / \mathrm{MM}$ optimisations. The simulations were run using the ChemShell interface ${ }^{43}$ employing Turbomole ${ }^{44}$ for the QM part and 
the DL_poly ${ }^{45}$ routines included in ChemShell for the MM region with electronic embedding. The QM model consisted of the T1 Cu complex, including the $\mathrm{Cu}$ ion, His395, Cys453, Ile455, His458 and Phe463 The protein backbone was omitted, represented by a cut between $C_{\alpha}$ and $C_{\beta}$. Hydrogen atoms were used as link atoms in place of the $C_{\alpha}$, supported by a charge shift scheme. ${ }^{46}$ The active region for the simulation consisted of all residues with atoms within $12 \AA$ around the T1 Cu (about 1100 atoms, slightly varying between snapshots), excluding those amino acids and water/hydroxyl units that coordinated to the TNC cluster. The latter was to prevent artefacts to the structure from missing force field parameters as we could not carry the force field restraints from the MD simulation over to the $\mathrm{QM} / \mathrm{MM}$ approach due to restrictions in the employed software; the $\mathrm{QM} / \mathrm{MM}$ system as a whole however still contained all atoms from the MD simulation, with the atoms outside the active region being frozen.

For the QM region, we employed BP86/def2-SVP/def2-TZVP(Cu) and Charmm27 for the MM region, which contributes via its bonding/non-bonding MM-MM terms and the van-der-Waals QM-MM terms (forming $E_{M M}$ ). Three optimisations were performed for each snapshot: One for each redox state of $\mathrm{T} 1 \mathrm{Cu}$, namely $\mathrm{Cu}(\mathrm{I})$ and $\mathrm{Cu}(\mathrm{II})$, starting directly from the MD snapshot, and another $\mathrm{Cu}(\mathrm{I})$ optimisation starting from the $\mathrm{Cu}(\mathrm{II}) \mathrm{QM} / \mathrm{MM}$ optimised structure. The latter $\mathrm{Cu}(\mathrm{l})$ structures turned out to perform better in terms of the data set's standard deviation w.r.t. the average RP and are used to compute the RPs presented in the main article; the results for the directly computed $\mathrm{Cu}(\mathrm{I})$ geometries are presented in the SI.

\section{Single points for laccase redox potentials}

We performed single point calculations for each of the optimised QM/MM geometries with our "best candidate" DFT method (M06/6-311++G**/SDD(Cu)) to obtain the ARP of the $\mathrm{Cu}(\mathrm{I}) / \mathrm{Cu}(\mathrm{II})$ laccase redox pair. These results will be discussed primarily; BP86/6-311++G**/SDD(Cu) data will be shown for comparison. Gaussian09 was used for these purposes, as the technical procedure was analogous to the calibration scheme above. We employed the $\mathrm{QM} / \mathrm{MM}$ optimised geometries of each of the $\mathrm{QM} / \mathrm{MM}$ optimisations for each snapshot, including the corresponding point charge field. In contrast to the calibration, there is no thermochemical correction. Open-shell $\mathrm{Cu}$ (II) systems were treated at the unrestricted Kohn-Sham DFT level, with negligible spin contamination.

To compute the ARP, we had to correct for the MM environment energy, as the point charges have different positions in the $\mathrm{Cu}(\mathrm{I})$ and $\mathrm{Cu}(\mathrm{II})$ single points. The first step is to remove the point charges' self-interaction energy from the SCF energy reported in Gaussian09. We then added the DL_Poly $\left(\mathrm{E}_{\mathrm{MM}}\right)$ contribution from the corresponding optimised snapshot geometry, as there is some contribution to the total energy difference from the geometry of the environment. We thus obtain our corrected single point energies for either the oxidant or reductant via

$E_{\text {corr }}=E_{S C F}-E_{S e l f}+E_{M M}$

\section{Gradual deprotonation scheme}

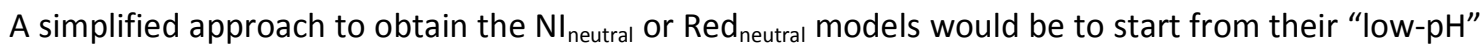
counterparts and just change the point charge field in the single point calculations without reoptimisation. This approach, while completely neglecting equilibration effects, allows for a qualitative and effective screening of the changes introduced by changes in the point charge field. ${ }^{4}$ 
Due to its low computational cost, we used this scheme to check for the effect of a gradual deprotonation. To do so, we have subjected all snapshots of the $\mathrm{Nl}_{\text {low-pH }}$ series to stepwise replacement of each of the 20 "adaptable" Glu or Asp residues. This was done by subsequently changing the next Glu/Asp candidate closest to the $\mathrm{T} 1 \mathrm{Cu}$ centre to its charged counterpart (by replacing the point charges of the neutral Glu or Asp by the charged versions). This way, we gradually converted the protein to its charged form, going from $\mathrm{NI}_{\text {low-pH }}$ to a $\mathrm{NI}_{\text {neutral }}$ mimic. To keep the overall system charge neutral, the $\mathrm{Cl}^{-}$ions closest to the candidate residue was deleted from the point charge field as well. The candidate Glu/Asp residues were, in order of deprotonation: Asp206, Asp456, Asp435, Glu460, Glu302, Asp167, Glu222, Asp444, Asp234, Asp50, Glu496, Asp101, Asp492, Asp23, Asp323, Asp470, Glu142, Asp364, Asp128 and Asp42. Note that the His98 residue was not adapted, as we aimed to see the effect from acidic residues only; His98 is also further away from the $\mathrm{T} 1 \mathrm{Cu}$ than any of the candidate acidic residues.

Since the simple deletion of charges in the point charge field introduces severe artefacts in the selfinteraction energy, the corresponding values need to be corrected before they can be used to compute the associated RPs (similar to Eq. (4)). The corrected energy for the modified point charge field (PCF) single points is

$$
\begin{aligned}
E(\text { Mod. PCF }) & =E_{S C F}(\text { Mod. PCF })-E_{\text {Self }}(\text { Orig. PCF })+E_{M M} \\
& =E_{\text {corr }}-E_{\text {Self }}(\text { Orig. } P C F)+E_{\text {Self }}(\text { Mod. PCF })
\end{aligned}
$$

Compared to a "real" physical system, Eqs. (5a) and (5b) are an approximation still; $\mathrm{E}_{\mathrm{MM}}$ contains spurious van-der-Waals and bonded terms for the modified amino acids. As we are however interested in only the effect from removing Coulomb interactions, E(Mod. PCF) is exactly what we require, since only the change in Coulombic self-interaction energy and $E_{S C F}$ enter into the calculation of the RP for the modified PCF.

\section{F463M mutant protein}

To generate the F463M mutant protein, the Phe residue was copied from the wild type, and its backbone segment was used to construct a Met residue using the molden software ${ }^{47}$ the Met residue was then inserted in place of the Phe463. Note that the corresponding MD restraints involving Phe463 had to be adapted accordingly; see SI.

\section{D206N mutant protein}

A D206N mutant protein was generated to predict the effect of an enforced neutral residue close to the T1 Cu site, in contrast to the Asp206 located in the wild type, which is expected to be neutral (i.e. protonated) only at low $\mathrm{pH}$, but negatively charged otherwise. To save computational effort, the mutant protein was generated on the basis of the optimised $\mathrm{NI}_{\text {low-pH }}$ model structure by simply replacing the $\mathrm{CO}_{2} \mathrm{H}$ group of the protonated Asp206 by $\mathrm{CONH}_{2}$. The construct was then fully optimised and continued from there just as all other models ( $M D, Q M / M M$, single points).

\section{Results and Discussion}

Method calibration 
Table 1 RPs of D-/D model complexes (in V), computed with various density functionals

\begin{tabular}{|l|l|l|l|}
\hline & $E_{\text {ARP }}\left(\mathrm{D}^{-} / \mathrm{D}\right)$ & $\mathrm{E}_{\text {ARP }}\left(\mathrm{Fc} / \mathrm{Fc}^{+}\right)$ & $\mathrm{E}_{\text {RRP }}$ \\
\hline BP86 & 3.69 & 5.15 & -1.46 \\
\hline PBE & 3.57 & 5.08 & -1.51 \\
\hline PBE0 & 3.61 & 5.55 & -1.94 \\
\hline M06L & 3.45 & 5.00 & -1.56 \\
\hline M06 & 3.91 & 5.11 & -1.20 \\
\hline B3LYP & 3.64 & 5.55 & -1.92 \\
\hline WB97XD & 2.70 & 5.71 & -2.01 \\
\hline CAM-B3LYP & 3.71 & 5.73 & -2.01 \\
\hline B3PW91 & 3.67 & 5.60 & -1.93 \\
\hline Experiment ${ }^{22}$ & & $-0.98^{\mathrm{a}}$ \\
\hline
\end{tabular}

The results of our DFT test calculations with the D/D redox couple can be found in Table 1 . A more detailed version is given in the SI. We find the M06 method to perform best out of all tested methods and thus chose that functional for the study of the laccase RPs.

Redox potentials of laccase in low-pH and neutral environments

The computed $E_{A R P}$ values of protonated laccase are given for ten snapshots in Table 2. It can be seen that the $E_{A R P}$ is dependent on the actual snapshot; within a single set we can see an increased $\mathrm{E}_{\mathrm{ARP}}$ of $6.11 \mathrm{~V}$ compared to an average value $\mathrm{e}^{\S}$ of $5.37 \mathrm{~V}$ (for $\mathrm{NI}_{\text {low-pH }}$ with M06). Although the standard deviations are notable $(0.2 \mathrm{~V}-0.3 \mathrm{~V})$, such extreme deviations can be considered rare. Note that the standard deviation is slightly larger when directly optimising the $\mathrm{Cu}(\mathrm{I})$ structure from the MD snapshot as outliers become more frequent; see SI for details. Table 2 only presents ARPs for which the $\mathrm{Cu}(\mathrm{I})$ geometry was optimised starting from the $\mathrm{Cu}(\mathrm{II})$ minimum.

Table $2 \mathrm{~T} 1$ centre $\mathrm{E}_{\mathrm{ARP}}$ (in V) of laccase in low-pH environment, for $\mathrm{NI}$ and Red TNC

\begin{tabular}{|c|c|c|c|c|}
\hline \multirow[b]{2}{*}{ Time } & \multicolumn{2}{|l|}{$\mathrm{NI}_{\text {low-pH }}$} & \multicolumn{2}{|l|}{$\operatorname{Red}_{\text {low-pH }}$} \\
\hline & BP86/MM & $\mathrm{M} 06 / \mathrm{MM}^{\mathrm{a}}$ & BP86/MM & ${\mathrm{M} 06 / \mathrm{MM}^{2}}^{2}$ \\
\hline $100 \mathrm{ps}$ & 4.92 & 5.45 & 4.69 & 5.25 \\
\hline $200 \mathrm{ps}$ & 4.63 & 5.17 & 4.42 & 4.99 \\
\hline $300 \mathrm{ps}$ & 4.71 & 5.23 & 4.58 & 5.13 \\
\hline $400 \mathrm{ps}$ & 4.48 & 5.03 & 5.14 & 5.68 \\
\hline $500 \mathrm{ps}$ & 4.68 & 5.22 & 4.52 & 5.13 \\
\hline $600 \mathrm{ps}$ & 4.62 & 5.19 & 4.69 & 5.24 \\
\hline $700 \mathrm{ps}$ & 5.09 & 5.63 & 4.35 & 4.91 \\
\hline $800 \mathrm{ps}$ & 4.99 & 5.48 & 4.55 & 5.11 \\
\hline 900 ps & 5.59 & 6.11 & 4.56 & 5.12 \\
\hline $1000 \mathrm{ps}$ & 4.65 & 5.19 & 4.50 & 5.05 \\
\hline Average & 4.83 & 5.37 & 4.60 & 5.16 \\
\hline Std. Dev. & 0.31 & 0.30 & 0.20 & 0.20 \\
\hline Avg. $E_{R R P}^{b}$ & -0.32 & 0.26 & -0.55 & 0.05 \\
\hline$\Delta$ Avg.(NI-Red) & 0.23 & 0.21 & Exp. RRP ${ }^{48}$ & $-0.15^{b, c}$ \\
\hline
\end{tabular}

${ }^{a}$ Using the BP86 QM/MM geometry ${ }^{b}$ vs. $\mathrm{Fc} / \mathrm{Fc}^{+c}$ shifted from $\mathrm{SHE}$ to $\mathrm{Fc} / \mathrm{Fc}^{+}$ 
It is difficult to compare the values in Table 2 to previous work. For a slightly smaller QM model, Hong and co-workers report an $\mathrm{E}_{\mathrm{ARP}}$ of $5.00 \mathrm{~V}$ (PBE/6-31G*), without explicitly stating details about the protein protonation state or the TNC charge composition. ${ }^{8} \mathrm{Li}$ and co-workers provide redox potentials of the T1 site depending on the TNC charge state; they state that the TNC state can affect the RP of the T1 site by up to $0.2 \mathrm{~V}$ (B3LYP-D3/def2-TZVPD). ${ }^{9}$ In the specific case of NI vs. Red studied here, with both TNCs displaying a net charge of +2 , they find however only a limited effect, $0.43 \mathrm{~V}$ (NI) compared to 0.41 to $0.47 \mathrm{~V}$ (Red) resulting only in a -0.02 to $+0.04 \mathrm{~V}$ change in RP. Our results indicate the effect to be $0.22 \mathrm{~V}$ in the M06 case and $0.24 \mathrm{~V}$ for BP86; the difference between the two papers is however close to our standard deviations of the individual absolute RPs. Methodologically, $\mathrm{Li}$ and co-workers have employed a scheme that includes a much stronger sampling of the MM part, with frozen QM core, hence the origins for their lower range might lie here. We provide a small test for our sampling method in the SI (Table S30), showing random smaller sample sets change the ARPs only by about $0.1 \mathrm{~V}$. The difference between $\mathrm{NI}$ and Red is even more pronounced when the protein protonation state is set up for a neutral environment (Table 3, see below for further discussion), 0.29 $\checkmark$ for M06 (0.31 V for BP86). Since the model employed by Li et al. also contains the TNC in an explicit $2 \mathrm{QM}$ model, it remains to be seen if the differences arise from methodological or physical reasons. We however share their assessment that the main TNC/T1 interaction arises from Coulomb forces, since no other relevant long-range interactions are possible in our model.

As seen during the method calibration (Table 1), the BP86 results tend to underestimate the redox potential compared to experiment, and Table 2 shows that this is the case for the laccase model as well. It is not fully clear which precise oxidation state of the TNC the experimental RRP refers to; while the NI state likely corresponds to the most oxidising state, and thus to the experimentally reported maximum RP, an experimental RP of the Red state is not as easily found. M06 seems to overestimate the potential noticeably, by about 0.41 or $0.20 \mathrm{~V}$ for $\mathrm{NI}$ or Red, respectively, while it underestimated the RRP of the D/D complex. To understand this difference, we have to look at the $\mathrm{pH}$-dependence in more detail. The results in Table 2 have been obtained for a low-pH model, assuming all titratable residues are protonated. We have also attempted to model a neutral environment, changing the protein protonation pattern to that expected for $\mathrm{pH} 7$ (see computational details), which results in significant changes in T1 RP (compare data in Tables 2 and $3)$. 
In the neutral case, we find M06 to predict the T1 RRP to be $-1.07 \mathrm{~V}(\mathrm{NI})$ or -1.34 (Red). Incidentally these values are quite close to the result for the $D^{\top} / D$ model $\left(E_{R R P}=-1.20 \mathrm{~V}\right)$. We can thus conclude that the RP of the T1 site is very sensitive to the overall laccase charge and that the large increase in the RRP observed on going from such a molecular model to the active enzyme is related to the capability of the latter to concentrate positive charges. The perfect, full protonation as used for the generation of Table 2 is an extreme case for which all possible residues have been neutralised. This is an unrealistic situation, as the protonation under acidic conditions is, arguably, not $100 \%$ complete at all times. The same can be said for the "neutral" environment models. Consequently, the actual RP will be in between the average RPs shown in Table 2 and Table 3. Table 2 thus represents the upper boundary for the laccase T1 RP (at the M06 level), while Table 3 displays the results for an inactive protein in a neutral environment. Fully neutralised, the protein displays a much lower RP, comparable to the RP of the pristine $D^{\circ} / D$ model complex without a protein environment. Unfortunately, we were unable to find any experimental $\mathrm{pH}$-dependence data on the RP for $T$. versicolor laccase; however, experiments for other laccases are available. They show that the RP drops slightly when going from harsh, low-pH conditions to the neutral regime, e.g. by $0.16 \mathrm{~V}$ for Myceliophthora thermophila laccase. ${ }^{49}$ While the results agree qualitatively, our drop of up to $1.4 \mathrm{~V}$ (M06 data) is one order of magnitude higher than the one found in experiment for Myceliophthora laccase. The precise reason for this apparent discrepancy is unclear at the moment. There might be conformational changes in the protein upon $\mathrm{pH}$ change that are not or not fully accounted for in our protocol, which might affect the RRP. It might also be that assuming a single static protonation state at $\mathrm{pH} 7$ is too simplistic, and that a dynamic equilibrium would have to be considered, i.e. involving proteins with the same overall charge, but different distributions of the protons over the individual residues (see also footnote §).

Table $3 \mathrm{~T} 1$ centre $\mathrm{E}_{\mathrm{ARP}}$ (in $\mathrm{V}$ ) of laccase in neutral environment, for $\mathrm{NI}$ and Red TNC

\begin{tabular}{|c|c|c|c|c|}
\hline \multirow[b]{2}{*}{ Time } & \multicolumn{2}{|l|}{$\mathrm{NI}_{\text {neutral }}$} & \multicolumn{2}{|l|}{ Red $_{\text {neutral }}$} \\
\hline & BP86/MM & $\mathrm{M06} \mathrm{MM}^{\mathrm{a}}$ & BP86/MM & $\mathrm{M06} / \mathrm{MM}^{\mathrm{a}}$ \\
\hline $100 \mathrm{ps}$ & 3.74 & 4.20 & 3.01 & 3.52 \\
\hline $200 \mathrm{ps}$ & 3.17 & 3.66 & 3.01 & 3.53 \\
\hline $300 \mathrm{ps}$ & 3.59 & 4.08 & 3.09 & 3.60 \\
\hline $400 \mathrm{ps}$ & 3.57 & 4.03 & 3.22 & 3.74 \\
\hline $500 \mathrm{ps}$ & 3.53 & 4.03 & 3.37 & 4.20 \\
\hline $600 \mathrm{ps}$ & 3.67 & 4.16 & 3.24 & 3.75 \\
\hline $700 \mathrm{ps}$ & 3.40 & 3.89 & 3.32 & 3.82 \\
\hline $800 \mathrm{ps}$ & 3.60 & 4.09 & 3.18 & 3.67 \\
\hline $900 \mathrm{ps}$ & 3.50 & 4.01 & 3.42 & 3.93 \\
\hline $1000 \mathrm{ps}$ & 3.76 & 4.29 & 3.43 & 3.94 \\
\hline Average & 3.53 & 4.04 & 3.23 & 3.77 \\
\hline Std. Dev. & 0.16 & 0.17 & 0.15 & 0.20 \\
\hline Avg. $E_{R R P}{ }^{b}$ & -1.62 & -1.07 & -1.92 & -1.34 \\
\hline$\Delta$ Avg.(NI-Red) & 0.30 & 0.27 & Exp. RRP ${ }^{48}$ & $-0.15^{b, c}$ \\
\hline
\end{tabular}




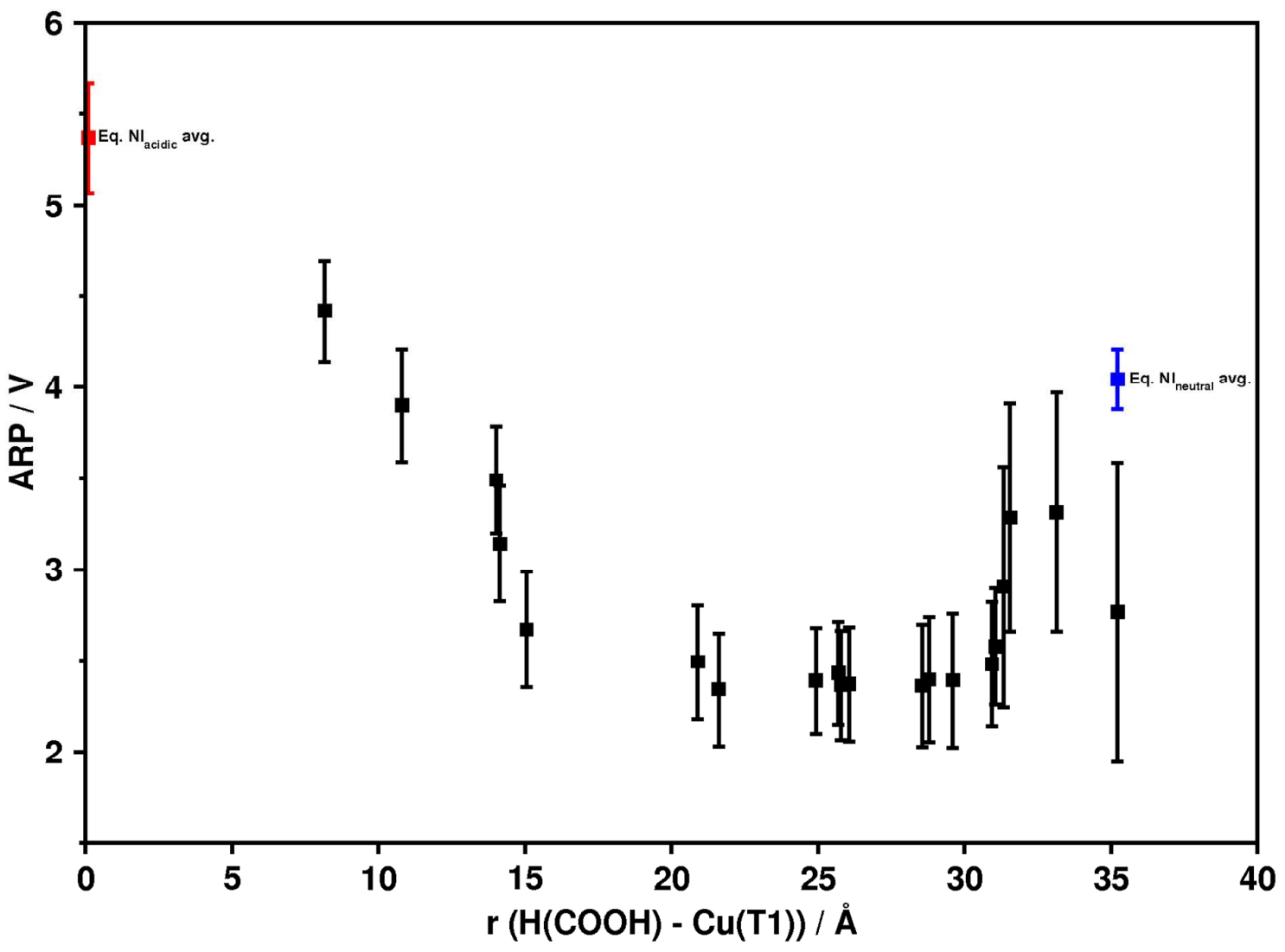

Figure $3 \mathrm{ARP}$ at different point charge field compositions, due to gradual replacement of uncharged Glu or Asp residues by their charged counterparts. Distance measured to the carboxylic proton in each Glu or Asp residue, based on the 600 ps snapshot (arbitrary choice, standard deviation of $r$ is less than $0.1 \AA ̊$ over all snapshots, not shown). Corresponding ARPs from the equilibrated $\mathrm{QM} / \mathrm{MM}$ trajectories indicated for comparison. Error bars indicate the standard deviation over all 10 MD snapshots.

\section{Gradual deprotonation}

To understand the possible impact of individual amino acids on the RRP, we tried to mimic a stepwise deprotonation of the $\mathrm{NI}_{\mathrm{low}-\mathrm{pH}}$ model through a simple charge-adaption scheme in the $\mathrm{QM} / \mathrm{MM}$ single point calculations. For each step, the charges of a single pair of neutral acid/ $\mathrm{Cl}^{-}$in the point charge field were changed to those of to the corresponding negatively charged carboxylate/no counterion. The sequence of residues to be deprotonated was determined by distance from T1, starting from the nearest one. This charge adaption was carried out for all 10 snapshots. The averaged results can be seen in Figure 3, a display of the individual data series can be found in the SI.

As the charges of the nearest Asp206 at $8 \AA$ from T1 are mutated, a huge drop of the ARP is obtained, by nearly $1 \mathrm{~V}$ on average (on going from the first data point on the left to the second one in Figure 3). The extent to which this single residue is protonated in an equilibrium can thus have a large effect on the overall redox potential. Successive "deprotonation" further reduces the computed ARP, which only starts to level off at about $2.4 \mathrm{~V}$ for distances between 15 to $30 \AA \AA$. In our study of the redox potential of lignin peroxidase we had noted similarly long-range effects upon deleting charges of individual amino acid residues. ${ }^{450}$ As more charges beyond $30 \AA$ are introduced, i.e. closer to the periphery of the solvation sphere, the computed ARP increases again, as does its standard deviation. This scatter is probably related to the removal of the chloride charges that are 


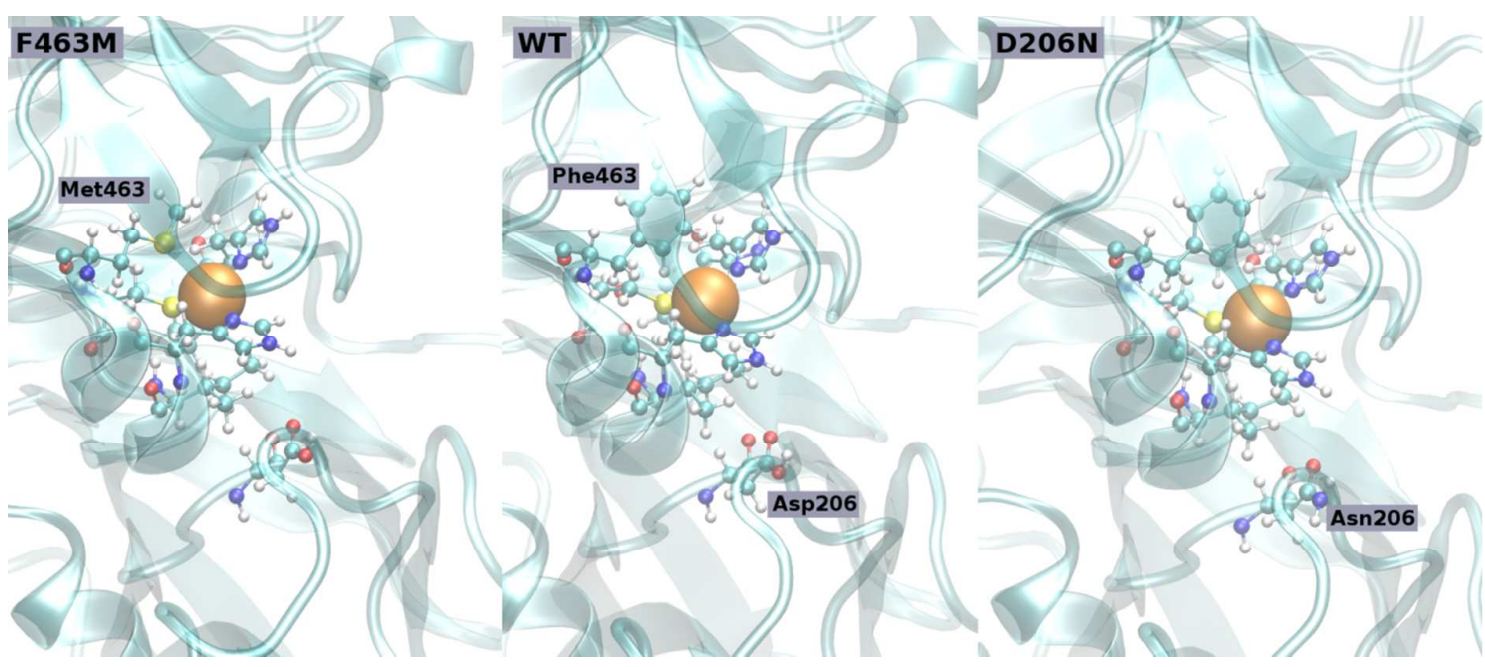

Figure 4 Investigated mutant structures compared to the wild type (WT). Force field optimised structures shown.

actually not close to the amino acids in question, and the dearth of remaining $\mathrm{Cl}^{-}$candidates for this purpose. As the $\mathrm{NI}_{\text {neutral }}$ and the fully converted point charge field at the right hand side of Figure 3 contain the same systems, the origin of their difference in ARP must be located in the geometry. The final ARP of the rightmost point in Figure 3 is $2.77 \mathrm{~V}$, significantly lower than the value of $4.04 \mathrm{~V}$ in Table $3\left(\mathrm{NI}_{\text {neutral }} / \mathrm{M} 06\right)$ for the equilibrated and relaxed structures; hence the effect of enzyme conformation can amount to at least $1.3 \mathrm{~V}$ in laccase. We can conclude that the dynamics and structural flexibility of laccase favours a high ARP, since a direct, "frozen" deprotonation would lead to a lower ARP.

\section{D206N mutant protein}

We have seen above that Asp206, the acidic residue closest to T1, needs to be in its protonated, neutral state to achieve a high redox potential. Arguably, a low pH is required for this, rationalising the requirement of acidic conditions for a high enzymatic activity. If this residue could be replaced with one that stays neutral irrespective of the $\mathrm{pH}$, a high oxidative power could be maintained also at higher $\mathrm{pH}$, which might enable lignin degradation under milder, less strongly acidic conditions. The D206N variant (Figure 4) would seem a good candidate for such a mutation, because it should appropriately keep the hydrogen bonding interactions intact, maintaining the structural integrity of the enzyme. We have prepared such a mutant protein, assuming the NI state of the T3 cluster and protonated residues as in the low-pH calculations (save for Asn206), for comparison with the wild type in its state of highest RP (Table 2). The corresponding RPs of the mutant protein can be found in Table 4.

Surprisingly, this exchange of a neutral residue for another does result in a noticeable change of the predicted redox potential, by about $-0.3 \mathrm{~V}$. Being of the same order as the standard deviations obtained for each protein, this change is barely significant, although the average RPs for our wild type proteins are robust within $0.1-0.2 \mathrm{~V}$ when taken from smaller sample sizes (see SI). Because the prediction that a high redox potential would be maintained through this mutation can be made only at a low confidence level, the origin of this computed change would be of interest. It is unlikely that the mutation would induce a significant change in the distance between the T1 $\mathrm{Cu}$ and the exchanged amino acid, since Asn and Asp have rather similar hydrogen bonding capabilities. In fact 
Table 4 T1 centre $E_{\text {ARP }}$ (in V) of D206N laccase in low-pH environment, for the NI TNC state

\begin{tabular}{|l|l|l|}
\cline { 2 - 3 } \multicolumn{1}{c|}{} & \multicolumn{2}{l|}{$\mathbf{N I}_{\text {low-pH/D206N }}$} \\
\hline Time & BP86/MM & $\mathbf{M 0 6}_{\mathbf{M}} \mathbf{M M}^{\mathrm{a}}$ \\
\hline $100 \mathrm{ps}$ & 4.57 & 5.10 \\
\hline $200 \mathrm{ps}$ & 4.69 & 5.20 \\
\hline $300 \mathrm{ps}$ & 4.47 & 4.99 \\
\hline $400 \mathrm{ps}$ & 4.63 & 5.13 \\
\hline $500 \mathrm{ps}$ & 5.29 & 5.76 \\
\hline $600 \mathrm{ps}$ & 4.14 & 4.67 \\
\hline $700 \mathrm{ps}$ & 4.47 & 4.97 \\
\hline $800 \mathrm{ps}$ & 4.03 & 4.55 \\
\hline $900 \mathrm{ps}$ & 4.54 & 5.07 \\
\hline $1000 \mathrm{ps}$ & 4.29 & 4.82 \\
\hline Average & $\mathbf{4 . 5 1}$ & $\mathbf{5 . 0 3}$ \\
\hline Std. Dev. & $\mathbf{0 . 3 3}$ & $\mathbf{0 . 3 2}$ \\
\hline Avg. ERRP $^{\mathrm{b}}$ & $-\mathbf{- 0 . 6 4}$ & $\mathbf{- 0 . 0 8}$ \\
\hline
\end{tabular}

the corresponding $\mathrm{C}\left(\mathrm{CO}_{2} \mathrm{H}\right)-\mathrm{Cu}$ and $\mathrm{C}\left(\mathrm{CONH}_{2}\right)$ - $\mathrm{Cu}$ distances do not differ much between the wild type and D206N (less than $0.1 \AA$ on average, data not shown).

Comparing the dynamics of the wild type and D206N more closely, we noticed a subtle structural detail involving Asp456. This is the second-closest Asp residue to the $\mathrm{T} 1 \mathrm{Cu}$ centre, and as such also very important for the electrostatics. In the mutant protein, the neutral protonated Asp456 binds exclusively to the backbone oxygen of Ser113 throughout the whole trajectory. For the wild type, however, there are two conformations present; the one binding to the backbone oxygen of Ser113 and one for which the residue binds to a crystal water oxygen (see Figure 5). The Ser113-bound conformation is slightly less populated in the set of our snapshots (4 out of 10), and incidentally corresponds to those snapshots (namely 400, 500, 600 and 1000 ps), for which we computed RPs

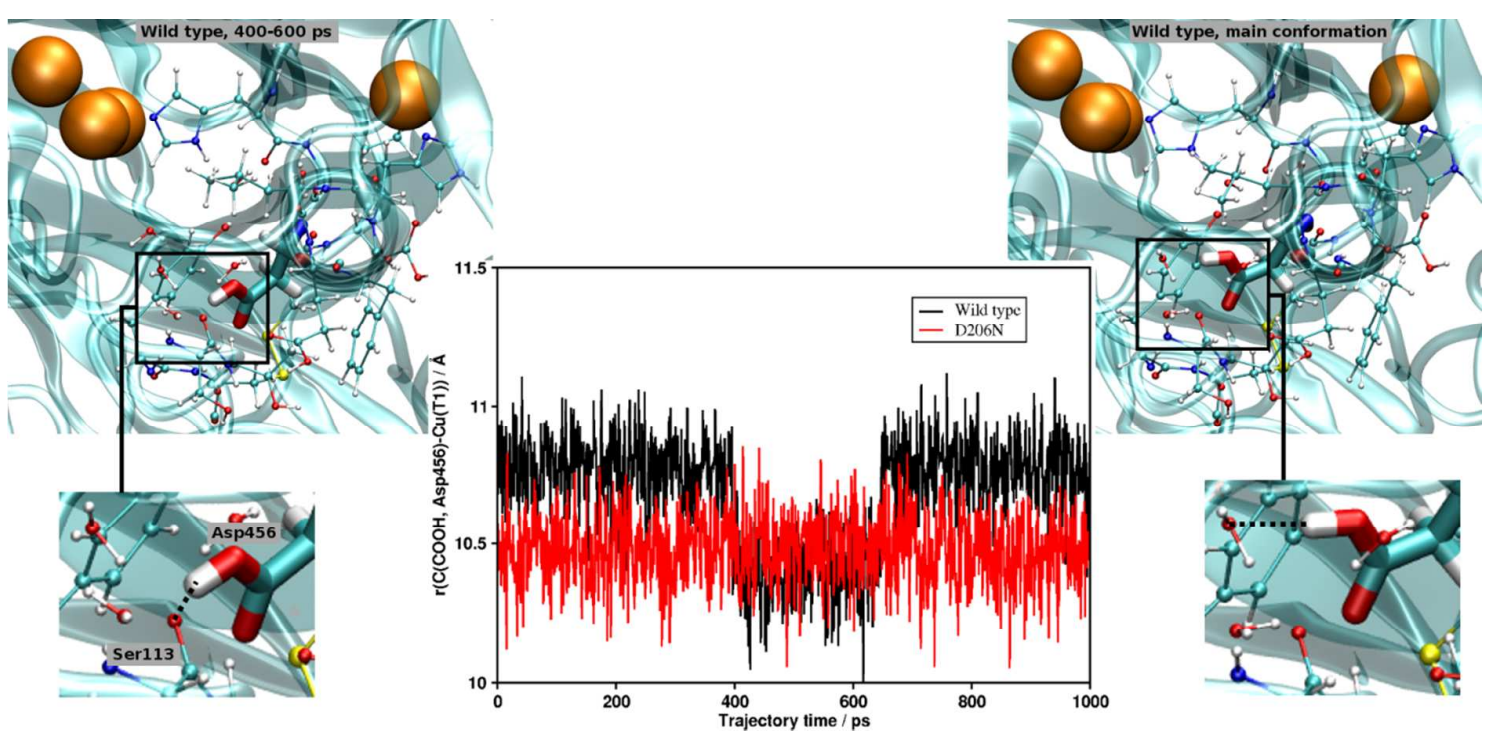

Figure 5 Difference between the Asp456 conformations in the wild type and D206N mutant laccase. Left: "Switched" conformation that is only temporarily present in the wild type, but always for the mutant (not shown). Asp456 binds to the backbone of Ser113. Centre: Distance plot between T1 Cu and Asp256, along the full MD trajectories of the wild type and the mutant. Right: Main conformation for the wild type Asp456, binding to a crystal water. 10 ns trajectories can be found in the SI. 
that are closer to the D206N average RP than most other wild type RPs. In this conformation Asp456 is slightly closer to the T1 Cu than in the other one (by ca. $0.25 \AA$ ). As such, one can speculate that the change in Asp456 hydrogen bonding might correlate with the T1 Cu RP, and the fact that D206N does not show any non-Ser113-bound conformations could rationalise the difference in RP. For instance, this conformational change could induce subtle differences in distances between the $\mathrm{Cu}$ centre and the charged residues (which are, arguably, more important for the RP than the neutral ones). As there are quite a few of these, however, it is difficult to disentangle their effect, and we cannot provide a conclusive explanation at this point for the subtle differences between the RPs of the D206N mutant protein and the wild type. We have checked for any further significant distance changes to T1 $\mathrm{Cu}$ for charged groups within $15 \AA$ of the T1 Cu centre; however all candidates are either remaining within the same distance regime $(<0.1 \AA$ difference in distance to T1 Cu for Arg161 and Asp424, data not shown), or their change in position does not correlate well to the observed RP (Arg121, see SI). Also it remains unclear how the D206N mutant would result in this "locked" Ser113Asp456 hydrogen bond. It might very well be that the mutant exhibits multiple conformations as well, but just does not show them on the time scale of our simulation. These uncertainties notwithstanding, Ser113 might be an interesting target for mutation, although it is not trivial to affect the backbone structure of a protein without disrupting its overall structure.

\section{F463M mutant}

As a comparison to several other MCO variants, we investigated the F463M mutant of laccase (Figure 4). Met-variants of MCOs tend to exhibit a slightly lower RP (by about 0.09 to $0.1 \mathrm{~V}$ ) than MCOs with Phe. ${ }^{19,51,52}$ Because both residues are neutral, this is a more subtle effect than the protonation/deprotonation equilibria studied above. On the other hand, these residues are much closer to the T1 site, so close in fact that both are included in the respective QM regions. We only present results for a $\mathrm{NI}_{\text {low-pH }}$ variant, as this is the most active form of the protein. The computed ARP of the $\mathrm{NI}_{\text {low-pH }} \mathrm{F} 463 \mathrm{M}$ mutant is on average lower by $0.08 \mathrm{~V}$ (BP86: $0.14 \mathrm{~V}$ ) than the RP computed for the equivalent wild type (see Table 2). We also found a strong dependence on the Cu oxidation state for the $\mathrm{Cu}$-S(Met463) distance, which is, for the the equilibrated data set, $3.22+/-0.04 \AA$ for $\mathrm{Cu}(\mathrm{I})$ and $2.67+/-0.08 \AA$ for $\mathrm{Cu}(\mathrm{II})$. Even though the difference in RP is well within our standard deviations

Table 5 T1 centre $E_{\text {ARP }}$ (in V) of F463M laccase in low-pH environment, for the NI TNC state. For the directly optimised snapshot mutants, differences to the parent wild type snapshot are given, $\Delta W T=E_{A R P}(M u t)-E_{A R P}(W T)$

\begin{tabular}{|c|c|c|c|c|c|c|}
\hline \multirow[b]{2}{*}{ Time } & \multicolumn{2}{|c|}{$\mathrm{NI}_{\text {low-pH/F463M / equilibrated }}$} & \multicolumn{4}{|c|}{$\mathrm{NI}_{\text {low-pH/F463M }} /$ from $\mathrm{NI}_{\text {low-pH }}$} \\
\hline & BP86/MM & ${\mathrm{M} 06 / \mathrm{MM}^{\mathrm{a}}}^{\mathrm{a}}$ & BP86/MM & $\Delta \mathbf{W T}$ & $\mathrm{M06} \mathrm{MM}^{\mathrm{a}}$ & $\Delta \mathbf{W T}$ \\
\hline $100 \mathrm{ps}$ & 4.52 & 5.13 & 4.93 & 0.01 & 5.54 & 0.08 \\
\hline $200 \mathrm{ps}$ & 4.46 & 5.07 & 4.78 & 0.15 & 5.38 & 0.22 \\
\hline 300 ps & 4.61 & 5.23 & 4.72 & 0.02 & 5.33 & 0.09 \\
\hline $400 \mathrm{ps}$ & 4.45 & 5.07 & 4.55 & 0.07 & 5.17 & 0.14 \\
\hline 500 ps & 4.07 & 4.68 & 4.74 & 0.07 & 5.36 & 0.14 \\
\hline $600 \mathrm{ps}$ & 4.53 & 5.15 & 4.59 & -0.03 & 5.22 & 0.03 \\
\hline 700 ps & 4.47 & 5.09 & 4.90 & -0.20 & 5.50 & -0.13 \\
\hline $800 \mathrm{ps}$ & 4.94 & 5.57 & 5.19 & 0.20 & 5.75 & 0.27 \\
\hline 900 ps & 4.16 & 4.81 & 5.69 & 0.09 & 6.25 & 0.13 \\
\hline 1000 ps & 4.35 & 5.03 & 4.81 & 0.17 & 5.40 & 0.22 \\
\hline Average & 4.46 & 5.08 & 4.89 & 0.05 & 5.49 & 0.12 \\
\hline Std. Dev. & 0.12 & 0.23 & 0.32 & $0.11^{c}$ & 0.30 & $0.11^{c}$ \\
\hline Avg. $E_{R R P}^{b}$ & -0.69 & -0.03 & -0.26 & 0.05 & 0.38 & 0.12 \\
\hline
\end{tabular}

${ }^{\mathrm{a}}$ Using the BP86 QM/MM geometry ${ }^{\mathrm{b}}$ vs. $\mathrm{Fc} / \mathrm{Fc}^{+{ }^{c}}$ Standard deviation of $\Delta \mathrm{WT}$, not $\triangle \mathrm{WT}$ of deviations 
over all snapshots, it is in pleasing overall agreement with the expectation from experiment. When the mutation is effected in the QM/MM minima of the wild type, directly followed by reoptimisation without additional equilibration, a somewhat different result is obtained: For such a model, a $0.12 \mathrm{~V}$ higher RP is found for the mutant, compared to the wild type (0.05 V for BP86). Although the standard deviations exceed the expected change in RP between mutant and wild type, it can be seen from that the ARPs found for the directly optimised set are all higher than the one of the equilibrated set (Table 5). As replacing a rather large Phe by a smaller Met residue introduces a strong change for the protein environment, the importance of the dynamics can be rationalised. Apparently, a direct mutation model can lead to qualitative errors if the dynamics of the equilibration are omitted. With this in mind, the combination of $\mathrm{M06}$ and the employed optimisation protocols appear to be well suited to provide reliable predictions for changes in RPs, at least for this type of mutation under equilibrated conditions.

\section{Conclusions}

We have studied the redox potential of the MCO laccase T1 site, in the presence of two different TNC cluster charge states. In terms of the redox potential, the influence exerted by the TNC on the $\mathrm{T} 1$ site of laccases amounts to up to $0.3 \mathrm{~V}$ of the RP, depending on the chosen method and protein protonation state. The computed ARPs are in good agreement with the ones previously reported. ${ }^{8}$ The difference in RP compares well to other studies on other MCOs. ${ }^{9}$ For the calculation of the RRP of a $\mathrm{Cu}$ model complex with a similar coordination environment as T1, M06 outperforms several other standard QM methods. Compared to experiment, ${ }^{48}$ the computed RRPs suffer slightly from optimisation with BP86 and omitting thermal and zero-point corrections, introducing an error of about 0.2-0.3 $\vee$ for our reference compound. Combined with the standard deviation that we obtain from our calculations, we conclude that the given approach can provide qualitative insights into the changes of RPs between different states of the laccase model, up to a semi-quantitative level when it comes to the direct comparison between wild type and mutant systems. This allows a direct comparison to our previous study on lignin peroxidase. ${ }^{4}$

We also varied the protonation state of the laccase, addressing charges that are mostly located on the surface, mimicking the neutral (intracellular) environment, and the acidic (extracellular) environment for active laccase. We found that the activity of laccase is suppressed in a neutral environment due to the presence of several acidic amino acids, which lead to an overall strong negative surface charge of laccase at neutral $\mathrm{pH}$. In that state, laccase exhibits a predicted RRP vs. $\mathrm{Fc} / \mathrm{Fc}^{+}$of $-1.34 \mathrm{~V}$ at the M06 level, $1.39 \mathrm{~V}$ lower than our idealised, fully protonated active form. Compared to experimental observations for other laccases, this trend is qualitatively correct, however the magnitude of this change appears to be significantly overestimated. As $T$. versicolor laccase is supposed to be active in the acidic extracellular environment, this $\mathrm{pH}$ dependence might contribute to a protective deactivation mechanism inside the cell, but the actual computed value appears to be quite large.

To analyse the origin of this $\mathrm{pH}$-dependence, we devised a simple method to model the effect of a gradual deprotonation of the system. Starting from the idealised fully protonated state, we successively modified the point-charge fields from neutral, protonated acid residues to those of their anionic carboxylates, without any geometry relaxation or equilibration, until the $\mathrm{pH}$-neutral state was reached. We found that the stepwise deprotonation converges at about $20 \AA$ distance from the 
T1 centre with an ARP of about $2.4 \mathrm{~V}$, which is far lower than the equilibrated average for the neutral laccase, $4.04 \mathrm{~V}$. We conclude that amino acids further away than $20 \AA$ should not influence the laccase T1 RP to a large amount and/or systematically; we also find that the overall laccase geometry favours a high ARP state by compensating for the changes in amino acid protonation by up to $1.6 \mathrm{~V}$; considering the comparison to experiment, the actual value is likely higher. Even only changing the closest amino acid, Asp206, to its charged counterpart (first point of the series in Figure 3 ) introduces a change in RP that is larger than the change found over whole $\mathrm{pH}$ ranges for laccases in experiment. The structural dynamics of laccase thus appear to enhance the RP of the protein and mask the effect of the variable charges, even when they are as close as $8 \AA$ to the T1 site such as Asp206.

Based on this finding, we explored a mutation to remove or alleviate the $\mathrm{pH}$ sensitivity of the laccase activity (D206N). However, it appears that this mutation might result in a subtle change in the protein dynamics, locking the Asp456 residue in a conformation with a slightly lower RP. This interpretation requires further testing, but could be a lead for the design of further mutations with the aim to maintain or even increase the oxidative power of laccase without the requirement of strongly acidic reaction conditions.

Finally, we also studied the RP of a F463M laccase mutant protein on the same levels of theory, to probe if the adopted methodology can describe the change upon mutation of this residue. We were able to reproduce the experimental change in the RP of a related enzyme $(0.09-0.1 \mathrm{~V})^{19,51,52}$ very closely $(0.08 \mathrm{~V})$. We conclude that the calibration on a small model complex results in a choice of QM method that can predict the effect of mutations in a qualitative fashion. We thus have an appropriate toolset at hand to predict the effects of biotechnological modifications on laccase, on a not overly expensive level, opening new avenues for rational re-design of this important enzyme.

\section{Supporting information}

Detailed tables of DFT method calibration, Onsager solvation tests, force field terms and parameters, dynamics of surface charge locations (additional video file), dynamics of Arg121, effect of starting $\mathrm{Cu}(\mathrm{I})$ optimisation from MD snapshot or $\mathrm{Cu}(\mathrm{II})$ structure, a full comparison to PBE results, RPs from subsets of the evaluated $\mathrm{QM} / \mathrm{MM}$ set to support convergence, additional $10 \mathrm{~ns}$ of wild type and D206N mutant MD.

\section{Acknowledgements}

The authors are grateful for funding by the Engineering and Physical Sciences Research Council, grant "Clean catalysis for sustainable development" (Ref. EP/J018139/1). JPG is grateful to Dr. Luke Crawford for valuable discussions.

\section{Notes}

$\S$ In principle, the optimised $\mathrm{QM} / \mathrm{MM}$ energies could be used to determine relative populations of individual structures from different snapshots (for a weighted average of the ARP), or to identify the most favourable sites of protonation. However, in practice this is not possible with our methodology, because the individual minima have a different equilibration history and the total energies are dominated to a large extent by the individual electrostatic and $\mathrm{H}$-bonding interactions involving the 
many thousands of solvent molecules. Free-energy MD simulations would have to be performed for this purpose, which are, however, very expensive with our DFT/MM approach.

\section{References}

1 Wong, D. W. S.. Structure and Action Mechanism of Ligninolytic Enzymes. Appl. Biochem. Biotechnol. 2009, $157,174-209$.

2 Jones, S. M.; Solomon, E. I. Electron Transfer and Reaction Mechanism of Laccases. Cell. Mol. Life Sci. 2015, $72,869-883$.

3 Pollegioni, L.; Tonin, F.; Rosini, E. Lignin-degrading Enzymes. FEBS J. 2015, 282, 1190-1213.

4 Castro, L.; Crawford, L.; Mutengwa, A.; Götze, J. P.; Bühl, M. Insights into Structure and Redox Potential of Lignin Peroxidase from QM/MM Calculations. Org. Biomol. Chem. 2016, 14, 2385-2389.

5 Solomon, E. I.; Augustine, A. J.; Yoon, J. $\mathrm{O}_{2}$ Reduction to $\mathrm{H}_{2} \mathrm{O}$ by the Multicopper Oxidases. Dalton Trans. 2008, 3921-3932.

6 Wang, J. H.; Feng, J. J.; Jia, W. T.; Chang, S.; Li, S. Z.; Li, Y. X. Lignin Engineering Through Laccase Modification: a Promising Field for Energy Plant Improvement. Biotechnol. Biofuels, 2015, 8, 145.

7 Castro, L.; M. Bühl, M. Calculations of One-Electron Redox Potentials of Oxoiron(IV) Porphyrin Complexes. J. Chem. Theory Comput. 2014, 10, 243-251.

8 Hong, G.; Ivnitski, D. M.; Johnson, G. R.; Atanassov, P.; Pachter, R. Design Parameters for Tuning the Type 1 Cu Multicopper Oxidase Redox Potential: Insight from a Combination of First Principles and Empirical Molecular Dynamics Simulations. J. Am. Chem. Soc. 2011, 133, 4802-4809.

9 Li, J. L.; Farrokhnia, M.; Rulisek, L.; Ryde, U. Catalytic Cycle of Multicopper Oxidases Studied by Combined Quantum- and Molecular-Mechanical Free-Energy Perturbation Methods. J. Phys. Chem. B 2015, 119, 82688284

10 Li, J. L.; Farrokhnia, M.; Heimdal, J.; Shleev, S.; Rulisek, L.; Ryde, U. Reorganization Energy for Internal Electron Transfer in Multicopper Oxidases. J. Phys. Chem. B 2011, 115, 13111-13126.

11 Piontek, K.; Antorini, M.; Choinowski, T. Crystal Structure of a Laccase from the Fungus Trametes Versicolor at 1.90-Angstrom Resolution Containing a Full Complement of Coppers. J. Biol. Chem. 2002, 277, 3766337669.

12 Pierloot, K.; De Kerpel, J. O. A.; Ryde, U.; Olsson; M. H. M.; Roos, B. O. Relation Between the Structure and Spectroscopic Properties of Blue Copper Proteins. J. Am. Chem. Soc. 1998, 120, 13156-13166.

13 Penfield, K. W.; Gewirth; A. A.; Solomon, E. I. Electronic Structure and Bonding of the Blue Copper Site in Plastocyanin. J. Am. Chem. Soc. 1985, 107, 4519-4529.

14 Zhekova, M.; Seth, H.; Ziegler, T. Density Functional Theory Studies on the Structure and Electron Distribution in the Peroxide Intermediate of the Catalytic Cycle of Multicopper Oxidases. Can. J. Chem. 2013, 91, 847-858.

15 Chalupsky, J.; Neese, F.; Solomon, E. I.; Ryde, U.; Rulisek, L. Multireference Ab Initio Calculations on Reaction Intermediates of the Multicopper Oxidases. Inorg. Chem., 2006, 45, 11051-11059.

16 Lowery, M. D.; Guckert, J. A.; Gebhard, M. S.; Solomon, E. I. Active-Site Electronic Structure and Contributions to Electron Transfer Pathways in Rubredoxin and Plastocyanin - Direct versus Superexchange. J. Am. Chem. Soc. 1993, 115, 3012-3013.

17 Humphrey, W.; Dalke, A.; Schulten, K. VMD: Visual Molecular Dynamics. J. Mol. Graph. 1996, 14, 33-38.

18 Olsson, M. H. M.; Hong, G. Y.; Warshel, A. Frozen Density Functional Free Energy Simulations of Redox Proteins: Computational Studies of the Reduction Potential of Plastocyanin and Rusticyanin. J. Am. Chem. Soc. 2003, 125, 5025-5039

19 Durao, P.; Bento, I.; Fernandes, A. T.; Melo, E. P.; Lindley, P. F.; Martins, L. O. Perturbations of the T1 Copper Site in the CotA Laccase from Bacillus Subtilis: Structural, Biochemical, Enzymatic and Stability Studies. J. Biol. Inorg. Chem. 2006, 11, 514-526.

20 Zhao, Y.; Truhlar, D. G. The M06 Suite of Density Functionals for Main Group Thermochemistry, Thermochemical Kinetics, Noncovalent Interactions, Excited States, and Transition Elements: Two New Functionals and Systematic Testing of Four M06-Class Functionals and 12 Other Functionals. Theor. Chem. Acc. 2008, 120, 215-241.

21 Li, J.; Fisher, C. L.; Chen, J. L.; Bashford, D.; Noodleman, L. Calculation of Redox Potentials and pK ${ }_{a}$ Values of Hydrated Transition Metal Cations by a Combined Density Functional and Continuum Dielectric Theory. Inorg. Chem. 1996, 35, 4694-4702. 
22 Melzer, M. M.; Mossin, S.; Cardenas, A. J. P.; Williams, K. D.; Zhang, S. Y.; Meyer; K.; Warren, T. H. A Copper(II) Thiolate from Reductive Cleavage of an S-Nitrosothiol. Inorg. Chem. 2012, 51, 8658-8660.

23 M. J. Frisch, G. W. Trucks, H. B. Schlegel, G. E. Scuseria, M. A. Robb, J. R. Cheeseman, G. Scalmani, V. Barone, B. Mennucci, G. A. Petersson, et al., Gaussian 09, Revision B.01, Gaussian, Inc., Wallingford CT, 2010.

24 Becke, A. D. Density-Functional; Exchange Energy Approximation with Correct Asymptotic Bahavior. Phys. Rev. A 1988, 38, 3098-3100.

25 Perdew, J. P. Density-Functional Approximation for the Correlation Energy of the Inhomogeneous Electron Gas. Phys. Rev. B, 1986, 33, 8822-8824.

26 Bühl, M.; Kabrede, H. Geometries of Transition-Metal Complexes from Density-Functional Theory. J. Chem. Theory Comput. 2006, 2, 1282-1290

27 Perdew, J. P.; Burke, K.; Ernzerhof, M. Generalized Gradient Approximation Made Simple. Phys. Rev. Lett. 1996, 77, 3865-3868.

28 Perdew, J. P.; Burke, K.; Ernzerhof, M. Generalized Gradient Approximation Made Simple (vol 77, pg 3865, 1996). Phys. Rev. Lett. 1997, 78, 1396-1396.

29 Vazquez-Lima, H.; Guadarrama, P. Analysis of Structural Factors Related to Spectroscopic Data and Redox Potentials of CUT1 Models Through DFT Tools. Int. J. Quantum Chem. 2012, 112, 1431-1438.

30 Vazquez-Lima, H.; Guadarrama, P.; Martinez-Anaya, C. Geometric Distortions on a Three-Coordinated T1 Cu Site Model as a Potential Strategy to Modulate Redox Potential. A Theoretical Study. J. Mol. Model. 2012, 18, 455-466.

31 Adamo, C.; Barone, V. Toward Reliable Density Functional Methods Without Adjustable Parameters: The PBEO Model. J. Chem. Phys. 1999, 110, 6158-6170.

32 Weigend, F.; Ahlrichs, R. Balanced Basis Sets of Split Valence, Triple Zeta Valence and Quadruple Zeta Valence Quality for H to Rn: Design and Assessment of Accuracy. Phys. Chem. Chem. Phys. 2005, 7, $3297-$ 3305.

33 Weigend, F. Accurate Coulomb-Fitting Basis Sets for H to Rn. Phys. Chem. Chem. Phys. 2006, 8, 1057-1065.

34 Hariharan, P. C.; Pople, J. A. Influence of Polarization Functions on Molecular-Orbital Hydrogenation Energies. Theor. Chim. Acta 1973, 28, 213-222.

35 Dolg, M.; Wedig, U.; Stoll, H.; Preuss, H. Energy-Adjusted Ab Initio Pseudopotentials for the 1st-Row Transition Elements. J. Chem. Phys. 1987, 86, 866-872.

36 Barone, V.; Cossi, M. Quantum Calculation of Molecular Energies and Energy Gradients in Solution by a Conductor Solvent Model. J. Phys. Chem. A 1998, 102, 1995-2001.

37 Cossi, M.; Rega, N.; Scalmani G.; Barone, V. Energies, Structures, and Electronic Properties of Molecules in Solution With the C-PCM Solvation Model. J. Comp. Chem. 2003, 24, 669-681.

38 Connelly, N. G.; Geiger, W. E. Chemical Redox Agents for Organometallic Chemistry. Chem. Rev. 1996. 96, 877-910

39 Isse, A. A.; Gennaro, A. Absolute Potential of the Standard Hydrogen Electrode and the Problem of Interconversion of Potentials in Different Solvents. J. Phys. Chem. B 2010, 114, 7894-7899.

40 This value has been used in the study of many water oxidation catalysts, see e.g.: (a) Liao, R.-Z.; Karkas, M. D.; Lee, B.-L.; Akermark, B.; Siegbahn, P. E. M. Photosystem II Like Water Oxidation Mechanism in a Bioinspired Tetranuclear Manganese Complex. Inorg. Chem. 2015, 54, 342-351; (b) Marenich, A. V.; Ho, J.; Coote, M. L.; Cramer, C. J.; Truhlar, D. G. Computational electrochemistry: prediction of liquid-phase reduction potentials. Phys. Chem. Chem. Phys. 2014, 16, 15068- 15106.

41 For Trametes versicolor (for other laccases in the range between $0.34 \mathrm{~V}-0.79 \mathrm{~V}$ ), cf. reference 49.

42 B. R. Brooks, C. L. Brooks, A. D. Mackerell, L. Nilsson, R. J. Petrella, B. Roux, Y. Won, G. Archontis, C. Bartels, M. Karplus, et al., CHARMM: The Biomolecular Simulation Program. J. Comp. Chem. 2009, 30, 1545-1614.

43 Sherwood, P.; de Vries, A. H.; Guest, M. F.; Schreckenbach, G.; Catlow, C. R. A.; French, S. A.; Sokol, A. A.; Bromley, S. T.; Thiel, W.; Turner, A. J.; et al. QUASI: A General Purpose Implementation of the QM/MM Approach and its Application to Problems in Catalysis. Theochem-J. Mol. Struct. 2003, 632, 1-28.

44 (a) Ahlrichs, R.; Bär, M.; Häser, M.; Horn, H.; Kolmel, C. Electronic-Structure Calculations on Workstation Computers - the Program System Turbomole. Chem. Phys. Lett. 1989, 162, 165-169; (b) Furche, F.; Ahlrichs, R.; Hättig, C.; Klopper, W.; Sierka, M.; Weigend, F. Turbomole. WIREs Comp. Mol. Sci. 2014, 4, 91-100.

45 Smith, W.; Yong, C. W.; Rodger, P. M. DL_POLY: Application to Molecular Simulation. Mol. Simul. 2002, 28, 385-471.

46 Sherwood, P.; de Vries, A. H.; Collins, S. J.; Greatbanks, S. P.; Burton, N. A.; Vincent, M. A.; Hillier, I. H. Computer Simulation of Zeolite Structure and Reactivity Using Embedded Cluster Methods. Faraday Discuss. 1997, 106, 79-92. 
47 Schaftenaar, G.; Noordik, J. H. Molden: a Pre- and Post-Processing Program for Molecular and Electronic Structures. J. Comput.-Aided Mol. Des. 2000, 14, 123-134.

48 B. R. M. Reinhammar, Oxidation-Reduction Potentials of Electron Acceptors in Laccases and Stellacyanin. Biochim. Biophys. Acta 1972, 275, 245-249.

49 Xu, F.; Berka, R. M.; Wahleithner, J. A.; Nelson, B. A.; Shuster, J. R.; Brown, S. H.; Palmer, A. E.; Solomon, E. I. Site-Directed Mutations in Fungal Laccase: Effect on Redox Potential, Activity and pH Profile. Biochem. J. 1998, 334, 63-70.

50 Such simple charge deletion scheme have a long history, see e.g.: Bash, P. A.; Field, M. J.; Davenport, R. C.; Petsko, A.; Ringe, D.; Karplus, M. Computer Simulation and Analysis of the Reaction Pathway of Triosephosphate Isomerases. Biochemistry 1991, 30, 5826-5832.

51 Battistuzzi, G.; Bellei, M.; Leonardi, A.; Pierattelli, R.; De Candia, A.; Vila, A. J.; Sola, M. Reduction Thermodynamics of the T1 Cu Site in Plant and Fungal Laccases. J. Biol. Inorg. Chem. 2005, 10, 867-873.

52 Xu, F.; Palmer, A. E.; Yaver, D. S.; Berka, R. M.; Gambetta, G. A.; Brown, S. H.; Solomon, E. I. Targeted Mutations in a Trametes Villosa Laccase - Axial Perturbations of the T1 Copper. J. Biol. Chem. 1999, 274, 12372-12375. 
22

Table of Contents entry

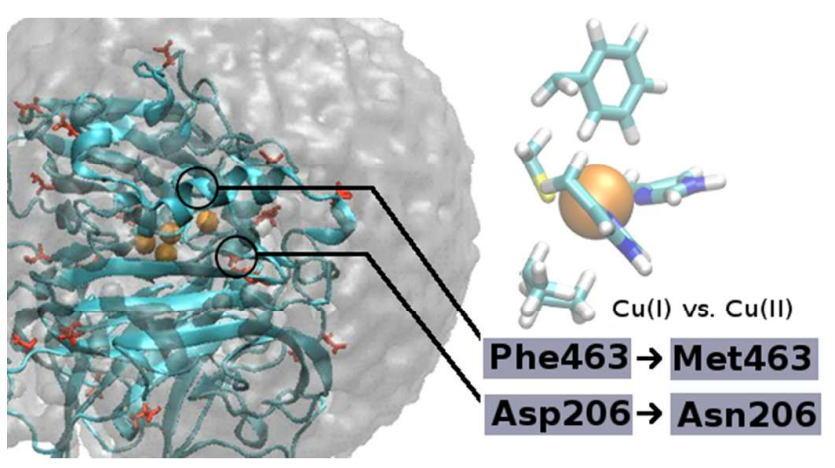

